Bundesgesundheitsbl 2014 $\cdot 57: 285-287$

DOI 10.1007/s00103-013-1917-9

Online publiziert: 20. Februar 2014

(c) Springer-Verlag Berlin Heidelberg 2014

\author{
U. Walter ${ }^{1}$ J. Töppich ${ }^{2}$ \\ ${ }^{1}$ Institut für Epidemiologie, Sozialmedizin und Gesundheitssystemforschung, \\ Medizinische Hochschule Hannover \\ ${ }^{2}$ Bundeszentrale für gesundheitliche Aufklärung (BZgA), Köln
}

\title{
Krebsfrüherkennung im Wandel
}

Mit dem am 9. April 2013 in Kraft getretenen „Gesetz zur Weiterentwicklung der Krebsfrüherkennung und zur Qualitätssicherung durch klinische Krebsregister (Krebsfrüherkennungs- und -registergesetz - KFRG)“ erfolgt in Deutschland eine politische Neuorientierung der Krebsfrüherkennung entsprechend den Empfehlungen der EU und WHO. Vorbereitet wurde diese durch den 2008 vom Bundesministerium für Gesundheit, von der Deutschen Krebsgesellschaft, der Deutschen Krebshilfe und der Arbeitsgemeinschaft Deutscher Tumorzentren initiierten Nationalen Krebsplan, in dem alle einschlägigen Einrichtungen und zahlreiche Experten verschiedener Disziplinen eingebunden waren und sind.

In der Januarausgabe 2014 des Bundesgesundheitsblattes wurde ein aktueller Überblick über Krebsregister und ihre Nutzung gegeben. Das vorliegende Schwerpunktheft richtet nun den Blick auf die Krebsfrüherkennung. Bereits im vergangenen Jahrzehnt wurde ein flächendeckendes Mammographie-Screening mit regelmäßiger Einladung eingeführt. Das KFRG sieht vor, innerhalb von 3 Jahren die derzeit als opportunistisch angebotenen Früherkennungsuntersuchungen für Zervixkarzinom und Darmkrebs zu bevölkerungsbezogenen und organisierten Krebsfrüherkennungsprogrammen auszubauen. Dieser Ausbau soll einhergehen mit der Bereitstellung von umfassenden und ausgewogenen Informationen über die Angebote der Krebsfrüherkennung und schließt eine kontinuierliche Qualitätssicherung mit systematischer Dokumentation, Monitoring und Evaluation ein. In der Um- setzung sind zahlreiche Fragen zu Abgrenzung der Zielgruppen, einbezogenen Verfahren, Untersuchungsintervallen, risikoadaptierter Früherkennung bis hin zur adäquaten Information und Ansprache der Bevölkerung zu beantworten.

Krebsfrüherkennung ist immer noch ein kontrovers diskutiertes Feld. Deshalb bietet dieses Schwerpunktheft einen Überblick über den aktuellen Stand und die zukünftigen Herausforderungen in der Krebsfrüherkennung. Hierzu haben wir 3 Themenblöcke gebildet:

(I) Einleitend erfolgt eine Einordnung der aktuellen Entwicklungen in der Krebsfrüherkennung in den gesundheitspolitischen und gesetzlichen Rahmen.

(II) Sieben Beiträge setzen sich anschließend mit dem Nutzen und Schaden von Krebsfrüherkennung sowie ethischen Wertabschätzungen und methodischen Herausforderungen auseinander. Dabei spannen wir den Bogen bewusst breit: Neben gesetzlich verankerten Leistungen werden auch individuelle Gesundheitsleistungen, neben Screening für die Allgemeinbevölkerung auch spezifische Herausforderungen für Risikogruppen betrachtet.

(III) Als unverzichtbar für die zukünftige Krebsfrüherkennung gilt die informierte Entscheidung der teilnahmeberechtigten Personen. Sie setzt eine hinreichende und ausgewogene Information über Nutzen und Schaden voraus. Diesem Thema ist der letzte Block mit 5 Beiträgen gewidmet. Er gibt einen Einblick in Konzepte der Information und zeigt auf, inwieweit derzeitige Informationsmaterialien eine informierte Entscheidung unterstützen. 
Im ersten Beitrag zeigt $A$. Helou die Neuerungen durch das Krebsfrüherkennungs- und -registergesetz (KFRG) auf und gibt einen Einblick in die Bemühungen der vergangenen Jahre, zu einer konsensorientierten Weiterentwicklung in der Krebsfrüherkennung zu gelangen. Krebsfrüherkennung befindet sich im Spannungsfeld zwischen dem bevölkerungsmedizinischen Ziel einer möglichst hohen Teilnahmerate an Krebsfrüherkennungsuntersuchungen und der Stärkung der Versicherten und Patienten als gut informierte Nutzer des Gesundheitssystems. Die Abwendung von der ausschließlichen Steigerung der Inanspruchnahme kann als Paradigmenwechsel in der deutschen Gesundheitspolitik bezeichnet werden.

Teil II beginnt mit einem Einblick in die Praxis des opportunistischen Screenings für das Zervixkarzinom durch U. Seifert und S.J. Klug. Mit einer derzeitigen Dreijahresteilnahmerate von $74 \%$ ist diese in Deutschland mit Ländern vergleichbar, die ein organisiertes Screening aufweisen. In der niedrigen HPV-Prävalenz vermuten sie vor allem eine problematische Qualität der zytologischen Untersuchung. Die Autorinnen zeigen die Evidenz für verschiedene Verfahren (PAP-Abstrich, Dünnschichtzytologie, HPV-Test), ihre Vor- und Nachteile sowie erforderliche Weiterentwicklungen zu einem organisierten Screening auf.

Zur Früherkennung von Darmkrebs liegen mehrere, z. T. als kosteneffektiv geltende, nichtinvasive (Stuhltests) sowie endoskopische (u. a. Koloskopie, Sigmoidoskopie) und radiologische Verfahren (CT/MR-Kolonographie) vor. Ihre Evidenz sowie aktuelle Weiterentwicklungen thematisieren H. Brenner und M. Hoffmeister in ihrem Beitrag. In einem organisierten Screening sowie verbesserten nichtinvasiven Tests sehen sie ein hohes Potenzial zur Steigerung der Effektivität der Darmkrebsfrüherkennung.

K. Rhiem und R.K. Schmutzler verdeutlichen für die kleine Gruppe der Frauen mit familiärem Erkrankungsrisiko die besonderen Anforderungen an eine Brustkrebsfrüherkennung: die intensivierte multimodale Früherkennung. Sie umfasst einen früheren Beginn, engmaschigere Screeningintervalle und den
Einbezug verschiedener Verfahren (Magnetresonanztomographie, Mammasonographie, Röntgenmammographie). Aktuelle Auswertungen der seit 2005 erhobenen Daten unterstreichen den Nutzen kombinierter Verfahren, was zur Modifikation der bisherigen Empfehlungen führt.

Die hinsichtlich ihres Nutzens umstrittene und in Deutschland nicht gesetzlich verankerte PSA-basierte Früherkennung des Prostatakarzinoms nehmen M. Graefen, T. Schlomm, T. Steuber und G. Sauter in den Blick. Ausgehend von 2 großen Studien, heben sie ihren Nutzen hervor. Die von Kritikern des PSA-Tests akzentuierten Gefahren der Überdiagnostik und von Übertherapien können nach Auffassung der Autoren durch eine bessere Charakterisierung und Prognoseabschätzung der entdeckten Prostatakarzinome sowie einer Stratifizierung der Patienten mit einem klinisch indolenten Prostatakarzinom in aktiver Überwachung vermindert werden.

H.-H. Dubben analysiert die „5 methodisch besten" Studien im aktuellen Cochrane-Review zum ProstatakrebsScreening. Er kommt zu dem Ergebnis, dass diese Früherkennung zu keiner Verbesserung der Prostatakrebs- und Gesamtmortalität führt, jedoch zu Überdiagnosen und Überbehandlungen. Der Autor benennt grundsätzliche methodische Herausforderungen von Studien zur Krebsfrüherkennung und kommt für das Prostatakrebs-Screening zu dem Schluss, dass aufgrund methodischer Probleme eine Reduzierung der Mortalität auch zukünftig nicht nachweisbar sein wird.

G. Marckmann und J. in der Schmitten erörtern die prinzipielle Frage, unter welchen Voraussetzungen eine Krebsfrüherkennung aus ethischer Sicht empfohlen werden kann. Sorgfältig abgewogen werden sollten der - auf Basis prospektiver randomisierter Studien ermittelte - Nutzen und Schaden. Zentral ist ebenfalls die informierte Entscheidung über die Teilnahme. Als Belastung in der Diskussion sehen die Autoren institutionelle und individuelle Interessenkonflikte der beteiligten Experten. Sie plädieren dafür, auch die Einschätzungen der Betroffenen systematisch in die Bewertung einzubeziehen.
Viele Krebsfrüherkennungsuntersuchungen, für die noch keine abschließende Bewertung vorliegt, werden als individuelle, durch den Versicherten zu finanzierende Gesundheitsleistungen (IGeL) angeboten. K. Koch, S. Thomas und $J$. Windeler weisen darauf hin, dass auch bei den IGe-Leistungen eine angemessene Aufklärung erforderlich ist, um eine abgewogene individuelle Entscheidung treffen zu können. Hierzu zeigen sie relevante Aspekte zur Bewertung von Nutzen und Schaden auf und plädieren für eine sprachlich saubere Trennung der Begriffe Früherkennung und Vorsorge.

Den dritten Teil eröffnen M.P. Anders, E. Baumann und E.W. Breitbart mit der Vorstellung eines kommunikationstheoretischen Konzeptes zur Prävention von Hautkrebs. Dieses zielt sowohl auf Information der Zielgruppen über UV-schützendes Verhalten als auch über die Vorund Nachteile der Inanspruchnahme des gesetzlich verankerten Hautkrebs-Screenings.

Voraussetzung für eine informierte Entscheidung sind wissenschaftlich abgesicherte und für die Zielgruppe verständliche Informationen zu Nutzen und Schaden von Krebsfrüherkennungsangeboten. U. Walter, J. Töppich und B. Stomper stellen das Design und erste Ergebnisse eines Projektes vor, das erstmals systematisch Printmaterialien und Internetangebote zu Darmkrebsfrüherkennung aus Experten- und Nutzerperspektive bewertet und die gewonnenen Erkenntnisse für den Aufbau eines Informationsmoduls zur Darmkrebsfrüherkennung nutzt.

Anhand eines neu entwickelten Kriterienkataloges zeigen $M$. Dreier, B. Borutta, G. Seidel, I. Kreusel, J. Töppich, E.M. Bitzer, M.L. Dierks, U. Walter, dass in den bewerteten Informationsmaterialien mögliche Nutzen häufiger als mögliche Schäden benannt wurden, quantifizierte Angaben hierzu eher selten gemacht wurden und Informationen zur Darmkrebsfrüherkennung mehrheitlich immer noch mit einer Aufforderung zur Teilnahme verbunden sind. Damit werden sie den Anforderungen an eine informierte Entscheidungsgrundlage nicht gerecht.

$\mathrm{Ob}$ die vorliegenden Gesundheitsinformationen zur Darmkrebsfrüherken- 
nung als Entscheidungsunterstützung wahrgenommen werden, untersuchten G. Seidel, I. Kreusel, M. Dreier, B. Borutta, U. Walter und M.L. Dierks et al. in einer qualitativen Studie. Die Rezeption der Broschüren und Flyer variiert vor dem Hintergrund des Bildungsgrades der Rezipienten. Generell treffen die Materialien auf eine Bevölkerung, die das Thema Darmkrebsfrüherkennung mit einem Appell zur Teilnahme verbindet und erst lernen muss, Gesundheitsinformationen abzuwägen, um informiert entscheiden zu können.

Der abschließende Beitrag von G. Haack, M. Köster und J. Töppich stellt den Aufbau eines Informationsmoduls zur Darmkrebsfrüherkennung vor, das die Projektergebnisse von Dreier et al. und Seidel et al. aufgreift. Dieses Modul wird in das Frauengesundheitsportal der Bundeszentrale für gesundheitlichen Aufklärung (BZgA) integriert mit dem Ziel, Informationen ausgewogen und zielgruppenspezifisch bereitzustellen, um Voraussetzungen für eine informierte Entscheidung zu schaffen.

Wir wünschen allen Lesern und Leserinnen eine spannende Lektüre und Auseinandersetzung mit der im Wandel begriffenen Krebsfrüherkennung.

\section{Korrespondenzadresse

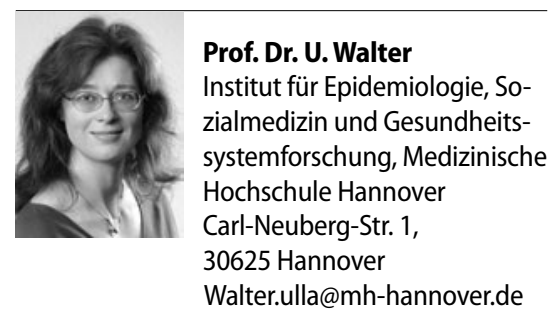

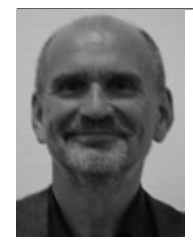

Dipl.-Soz.J. Töppich

Bundeszentrale für gesundheitliche Aufklärung (BZgA)

Ostmerheimerstr. 220,

51109 Köln

juergen.toeppich@bzga.de

Interessenkonflikt. U. Walter und J. Töppich geben an, dass kein Interessenkonflikt besteht.

\section{M.}

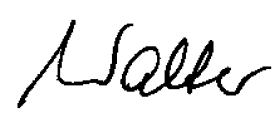

Ihre Ulla Walter

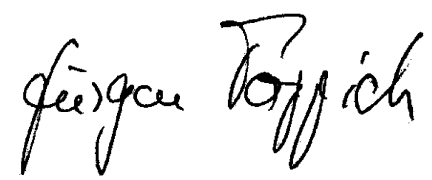

Ihr Jürgen Töppich 